\title{
ADSORÇÃO E PROPRIEDADES DE VOLUME DE MISTURAS BINÁRIAS ÁGUA ÁLCOOL: UM EXPERIMENTO DIDÁTICO COM BASE EM MEDIDAS DE TENSÃO SUPERFICIAL
}

\author{
Michelly C. dos Santos, Aline P. Moraes, Maykon A. Lemes, Emília C. D. Lima e Anselmo E. de Oliveira* \\ Instituto de Química, Universidade Federal de Goiás, CP 131, 74001-970 Goiânia - GO, Brasil
}

Recebido em 28/9/09; aceito em 2/3/10; publicado na web em 20/7/10

\begin{abstract}
AN UNDERGRADUATE EXPERIMENT IN PHYSICAL CHEMISTRY: ADSORPTION AND BULK PROPERTIES OF ALCOHOL-WATER MIXTURES BASED ON SURFACE TENSION MEASUREMENTS. An undergraduate physical chemistry experiment based on the drop counting method for surface tension measurements is proposed to demonstrate adsorption isotherms of binary aqueous solutions of ethanol, $n$-propanol, and $n$-butanol. Excess surface is obtained by the derivative of surface tension taken with respect to alcohol activity, after this activity calculation using van Laar equation. Laboratory class contents are surface tension, excess surface, percolation of hydrogen bonds, micelle, activity, and ideal solution.
\end{abstract}

Keywords: alcohol-water mixtures; adsorption isotherm; physical chemistry lab course.

\section{INTRODUÇÃO}

O projeto pedagógico do curso de Química do Instituto de Química da Universidade Federal de Goiás é fruto de inúmeras discussões coletivas e reflexões individuais. Foi concebido para ser o instrumento norteador não somente da prática pedagógica, mas também de reflexões acerca do contexto político e social em que se insere a Instituição, a região e o país. Para o atual cenário, o profissional da química deve possuir além de iniciativa, criatividade e adaptabilidade, um perfil com forte embasamento conceitual.

Dentro desse contexto, as disciplinas de química devem proporcionar o diálogo entre as atividades teóricas e práticas, ao invés de privilegiarem uma ou outra, visto que essas atividades são igualmente importantes para o futuro profissional. A formação do aluno do curso de Química, ou de outro curso cujas disciplinas de química compõem o projeto pedagógico, deve contemplar uma sólida formação teórica, além das habilidades características e requeridas no trabalho/ pesquisa em um laboratório de química, ${ }^{1}$ como o de Físico-Química Experimental.

Considerando os aspectos acima, um conteúdo importante em razão das aplicações dos processos que governam o comportamento físico químico dos líquidos é o de tensão superficial. O conceito de tensão superficial é central na compreensão dos fundamentos de química de coloides e de superfícies, aplicados em diversos setores industriais tais como os de tintas, ${ }^{2}$ detergentes, ${ }^{3}$ cosméticos, ${ }^{4}$ medicamentos, ${ }^{5}$ óleos lubrificantes, ${ }^{6}$ pesticidas, ${ }^{7}$ alimentos, ${ }^{8}$ além da importância em águas residuais de fábricas têxteis ${ }^{9}$ e em fenômenos da natureza. ${ }^{10}$

Desse modo, aulas práticas voltadas à determinação da tensão superficial de líquidos e à utilização dessa medida para determinar a concentração micelar crítica (CMC) de surfactantes e o excesso de superfície, bem como a interpretação dos resultados e a extensão da discussão para o aprofundamento do conhecimento da estrutura da matéria, em seus mais diversos aspectos, são fundamentais nas disciplinas de físico-química.

Alcoóis de baixa massa molar como metanol, etanol e $n$-propanol são completamente solúveis em água em qualquer faixa de composição. Já os alcoóis de maior massa molar, a começar pelo $n$-butanol, são parcialmente miscíveis em água. As soluções de água e álcool

\footnotetext{
*e-mail: elcana@quimica.ufg.br
}

são modelos representativos de desvio da idealidade em soluções aquosas, o que se evidencia pelas variações não lineares em suas propriedades físico-químicas e termodinâmicas. Por isso têm sido estudadas, por muito tempo, utilizando-se diversas ferramentas teóricas e experimentais como espectrometria de massa, ${ }^{11}$ difração de nêutrons, ${ }^{12}$ difração de raios-X,,$^{13}$ espectroscopia Raman ${ }^{14}$ e métodos de mecânica quântica ${ }^{15}$ e de dinâmica molecular. ${ }^{16}$ Esses estudos sustentam a hipótese de que não ocorre a solubilidade de alcoóis em água no nível molecular e que essas soluções são compostas de domínios (clusters) ricos em álcool e em água.

Em um artigo publicado no Journal of Colloid and Interface Science, em 2005, ${ }^{17}$ Yano apresenta as isotermas de adsorção de diversas misturas de água e álcool (metanol, etanol, propanol, 2-propanol, ter-butanol e $n$-butanol) calculadas usando dados de tensão superficial e pressão de vapor obtidos da literatura. A análise dos dados apresentados permite concluir que todos os sistemas apresentam um máximo na isoterma de adsorção, indicando a formação de uma monocamada de álcool na superfície. A formação da monocamada ocorre na concentração correspondente ao mínimo no volume molar parcial de excesso do álcool e à perda da idealidade da solução. Nessa concentração observa-se na curva de tensão superficial versus fração molar uma descontinuidade semelhante à observada em soluções de surfactantes na concentração micelar crítica, que é atribuída à perda da percolação das pontes de hidrogênio. Com base nessas observações, Yano sugere que medidas de tensão superficial de soluções água e álcool avaliadas em função da fração molar, bem como a construção das isotermas de adsorção com base nos valores calculados de excesso de superfície, permitem relacionar a estrutura na superfície e no seio da solução em misturas de água e álcool. Em soluções diluídas, as forças de hidratação hidrofóbicas induzem ao excesso de álcool na superfície. Quando a monocamada é completamente formada, as moléculas do álcool, tanto as da superfície quanto as do interior, ficam como que espremidas e começam a se agregar originando o desvio da idealidade da solução.

As informações contidas no artigo de Yano nos motivaram a propor essa aula, que emprega medidas de tensão superficial em soluções de água e álcool para mostrar a correlação estrutural entre a superfície do líquido e o seio da solução. São feitas determinações das tensões superficiais das soluções e essas medidas são utilizadas no cálculo do excesso de soluto na superfície, que é empregado na construção das isotermas de adsorção. 
Dentre as metodologias disponíveis para medidas de tensão superficial, a mais conveniente para ser empregada em aulas nos cursos de graduação, considerando o custo do aparato envolvido e a simplicidade na montagem do material prático, é o método da contagem do número de gotas, que é uma variante do método do peso da gota. ${ }^{18,19}$ No método do peso da gota, gotas que se desprendem da ponta de um tubo são coletadas até que a massa do líquido coletada, com base no volume que caiu, possa ser medida e usada para estimativa do peso da gota. Já no método da contagem, o número de gotas por volume fixo de solução é determinado.

O presente trabalho, obtido como resultado da aplicação prática em laboratórios de graduação do Instituto de Química da UFG, descreve uma proposta de aula de físico-química experimental, voltada ao ensino de alunos de graduação, para a determinação das isotermas de adsorção em misturas binárias de água e $n$-propanol. Essa proposta emprega uma metodologia experimental muito simples para demonstrar um conhecimento que já está bem documentado na literatura, em estudos por técnicas analíticas sofisticadas como espectroscopia de massa, difração de raios X, espectroscopia Raman e difração de nêutrons. A correlação estrutural entre a superfície do líquido e o seio da solução é obtida por meio das medidas da tensão superficial - método da contagem do número de gotas - e da aplicação da Equação de van Laar. Os resultados para os sistemas água etanol e água $n$-butanol também são apresentados como exemplos adicionais.

\section{PARTE EXPERIMENTAL}

O método da contagem do número de gotas baseia-se na Lei de Tate, ${ }^{20}$ onde o peso de uma gota que se desprende de um tubo é proporcional ao diâmetro do tubo e ao peso do líquido que sobe em um tubo, de mesmo diâmetro, por ação capilar. Como consequência, a razão entre as tensões superficiais de dois líquidos A e B, medidas com o mesmo tubo, resulta

$$
\frac{n^{A} \sigma^{A}}{\rho^{A}}=\frac{n^{B} \sigma^{B}}{\rho^{B}}
$$

Para cada líquido, $n$ representa o número de gotas por $\mathrm{mL}, \sigma$ a tensão superficial e $\rho$ a densidade. O resultado da Equação 1 mostra que a tensão superficial do líquido $A$ pode ser determinada quando se conhece a tensão superficial do líquido $B$. Uma vez determinadas as tensões superficiais das soluções, as isotermas de adsorção podem ser obtidas como resultado do gráfico do excesso superficial, $\Gamma$, em função da fração molar, ou do logaritmo dessa fração molar, de um dos componentes da mistura.

De acordo com a Equação de Gibbs para a adsorção na superfície, ${ }^{18}$ o excesso superficial do álcool em misturas água(2)-álcool(1), $\Gamma_{1}^{2}$, pode ser calculado por

$$
\Gamma_{1}^{2}=-\frac{a_{1}}{R T}\left(\frac{\partial \sigma}{\partial a_{1}}\right)_{T}
$$

com $a_{1}$ a atividade do álcool na solução, $T$ a temperatura em Kelvins e $R$ a constante dos gases. Nesse caso, resulta dessa equação que quando $\left(\frac{\partial \sigma}{\partial a_{1}}\right)_{T}$ é negativo, $\Gamma_{1}^{2}$ é positivo, com consequente excesso de soluto na superfície, e vice-versa. A atividade da mistura binária é obtida através da equação de van Laar, ${ }^{21}$ conforme a Tabela 13-3 da ref. 22.

$$
\ln \gamma_{1}=A_{12}\left(\frac{A_{21} x_{2}}{A_{12} x_{1}+A_{21} x_{2}}\right)^{2}
$$

onde $\gamma_{1}$ é o coeficiente de atividade, $x_{1}$ é a fração molar, e $A_{12}$ e $A_{21}$ são parâmetros de interação da mistura binária obtidos da Tabela 13-2 da ref. 22.

\section{Roteiro da aula}

O procedimento experimental é realizado com o preparo de 13 soluções aquosas de $n$-propanol conforme as composições da Tabela 1. Nesse ponto, o professor pode propor o estudo de outros sistemas como água etanol, ou água $n$-butanol, por exemplo, e decidir se toda uma turma estuda o mesmo sistema, ou se os diferentes sistemas serão estudados por grupos de alunos - esse último é preferível quando se tem limitação de solvente. Como caráter de atividade de experimentação investigativa, pode-se deixar que os próprios alunos escolham o sistema a ser estudado.

Um grupo de 4 alunos é dividido em 2, sendo que uma dupla determinará a densidade das soluções (com um picnômetro, por exemplo), enquanto que a outra dupla determina o número de gotas que caem em 1,0 mL de cada solução, da água e do álcool puro.

Após a conclusão da parte experimental procede-se ao tratamento dos dados - que pode ser iniciado no próprio laboratório de ensino, a depender da disponibilidade de computadores, ou ficar como atividade pós-aula, a ser avaliada em relatório de grupo. A vantagem do tratamento ser feito durante a aula é a possibilidade de discussão das etapas a serem feitas juntamente com o professor. Inicialmente, os alunos são questionados sobre a obtenção da isoterma. Conforme a Equação 2, esse resultado depende do comportamento da tensão superficial em função da atividade das soluções da mistura binária escolhida pelo grupo.

Em seguida, os alunos calculam os coeficientes de atividades das soluções com base na Equação da van Laar. Nesse ponto, cabe ressaltar que trabalhar com planilhas eletrônicas é uma habilidade indispensável para um futuro profissional. Um bom exemplo de software gratuito é o OpenOffice Calc, disponível no pacote BrOffice. ${ }^{23}$ O professor deve orientar a montagem da planilha para a realização dos cálculos. Uma sugestão de planilha consta no roteiro de aula proposta no Material Suplementar. Usando a relação $a=\gamma \cdot x$ são calculadas as atividades das soluções alcoólicas.

Após essa primeira parte de tratamento dos dados com planilha eletrônica e o cálculo das atividades, os alunos passam a interpretar os resultados obtidos com a construção dos gráficos da atividade e da tensão superficial em função da fração molar do álcool (ou em função do logaritmo neperiano dessa fração). Após o ajuste exponencial do gráfico da tensão superficial em função da fração molar, faz-se o ajuste exponencial (que também pode ser feito no próprio OpenOffice Calc). De posse dos resultados dessa função, e como o cálculo do excesso superficial é feito para cada valor de atividade, os alunos devem obter a tangente da curva em cada ponto. Nessa etapa, o conhecimento de derivadas parciais estudadas no curso de cálculo é empregado, de modo a resolver um problema prático. É importante ressaltar que esse conhecimento, por se tratar de um curso de nível superior, é mobilizado de modo a evitar que os resultados sejam obtidos por meio de retas traçadas com réguas. Além do mais, não se trata de uma derivada complexa, que necessite do aluno um conhecimento além do qual ele já se deparou em um curso de cálculo fundamental.

Tabela 1. Composição das soluções para o sistema água n-propanol

\begin{tabular}{lccccccccccccc}
\hline $\mathrm{n}$ & 1 & 2 & 3 & 4 & 5 & 6 & 7 & 8 & 9 & 10 & 11 & 12 & 13 \\
\hline $\mathrm{x}_{1} / 10^{-2}$ & 101 & 248 & 503 & 744 & 990 & 2,433 & 4,667 & 6,118 & 7,373 & 15,159 & 30,077 & 50,050 & 75,153 \\
\hline
\end{tabular}


Após o cálculo da derivada, os resultados são inseridos na Equação 2, e o excesso superficial é obtido para cada solução. A interpretação dos resultados da isoterma é feita após a obtenção do gráfico do excesso superficial em função do logaritmo neperiano da fração molar do álcool.

O tratamento dos dados é abordado a seguir, no tópico Resultados e Discussão. O procedimento experimental para a realização da aula é bastante simples, o que permite ao professor uma introdução aos conceitos envolvidos no desenvolvimento experimental. A parte de tratamento dos dados demanda um pouco mais de tempo, visto que depende da habilidade dos alunos com planilhas eletrônicas e com construção de gráficos. No entanto, percebe-se que alguns alunos já têm essa habilidade, o que não compromete o tratamento e a discussão dos resultados. Pode-se direcionar para que os alunos não se preocupem com a formatação dos resultados (letras em negrito, subescrito, etc.), o que possibilita um tempo maior para a discussão dos experimentos, sem que haja risco da prorrogação do tempo de realização da aula. É uma oportunidade para que o professor faça uma avaliação formativa, durante todo o processo de acompanhamento dos alunos, buscando subsídios para o melhoramento da prática pedagógica e do processo pedagógico. ${ }^{24}$ Essa metodologia facilita o emprego desse procedimento experimental em cursos com carga horária semanal menor do que $4 \mathrm{~h}$.

Outro ponto positivo da atividade dedicada ao tratamento dos dados é a aproximação dos alunos com o professor, fato facilitador do processo de ensino-aprendizagem, o que atende a um objetivo secundário da aula, visto que as isotermas são construídas como um resultado da troca de conhecimentos entre os alunos e o professor. A avaliação somativa feita com base nos relatórios mostrou que as isotermas obtidas pelos alunos estão de acordo com os resultados da literatura, ${ }^{17}$ além da mobilização de conceitos como atividade, coeficiente de atividade, tensão superficial, formação de agregados moleculares, adsorção, interface, derivadas parciais, e do desenvolvimento de habilidades como construção de gráficos, ajuste de função e manipulação de planilhas eletrônicas e handbook, por exemplo.

\section{RESULTADOS E DISCUSSÃO}

Os valores da tensão superficial para água(2) em função da temperatura podem ser obtidos na literatura, por consulta em um catálogo de propriedades físico-químicas como, por exemplo, a ref. 22; o número de gotas em 1,0 mL das soluções da Tabela 1 e da água pura, além das densidades medidas dos líquidos é usado na Equação 4 para a determinação da tensão superficial de cada uma das misturas binárias conforme

$$
\sigma_{\text {solução }}=\frac{n_{2} \sigma_{2}}{\rho_{2}} \times \frac{\rho_{\text {solução }}}{n_{\text {solução }}}
$$

Em seguida, são calculados os coeficientes de atividades das soluções com base na Equação de van Laar. Para o sistema água(2) $n$-propanol(1) os parâmetros da interação binária são $A_{12}=2,9095$ e $A_{21}=1,1572$. De posse dos coeficientes de atividade, são obtidas as atividades das soluções alcoólicas por $a=\gamma$. $x$. O comportamento dessas soluções pode ser visualizado pelo gráfico da atividade versus a fração molar do álcool, Figura 1, que evidencia um desvio positivo da solução ideal característico em soluções que apresentam interações intermoleculares via pontes de hidrogênio.

A taxa de variação da tensão superficial em função da atividade do álcool, $\left(d \sigma / d a_{1}\right)_{T}$, conforme a Equação 2, é obtida do gráfico da tensão interfacial versus a atividade, Figura 2. Esse comportamento é representado por um decaimento exponencial do tipo $\sigma=k e^{-a_{1} / k^{\prime}}+k^{\prime \prime}$, com $k, k$ ' e $k$ " os parâmetros de ajuste da função.

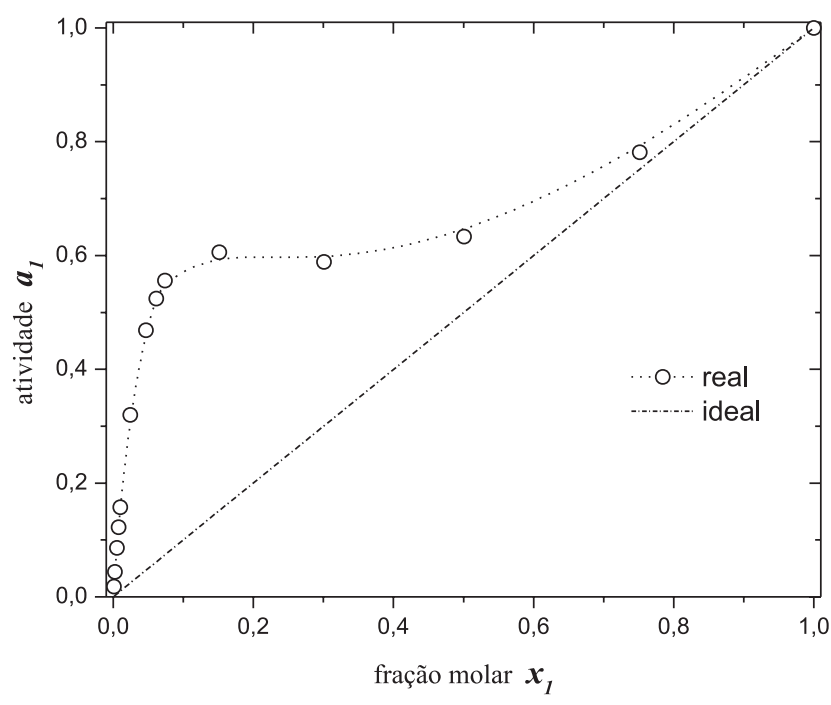

Figura 1. Gráfico da atividade, $a_{l}$, versus a fração molar de n-propanol, $x_{l}$, para o sistema água n-propanol. A linha reta tracejada reflete o comportamento ideal

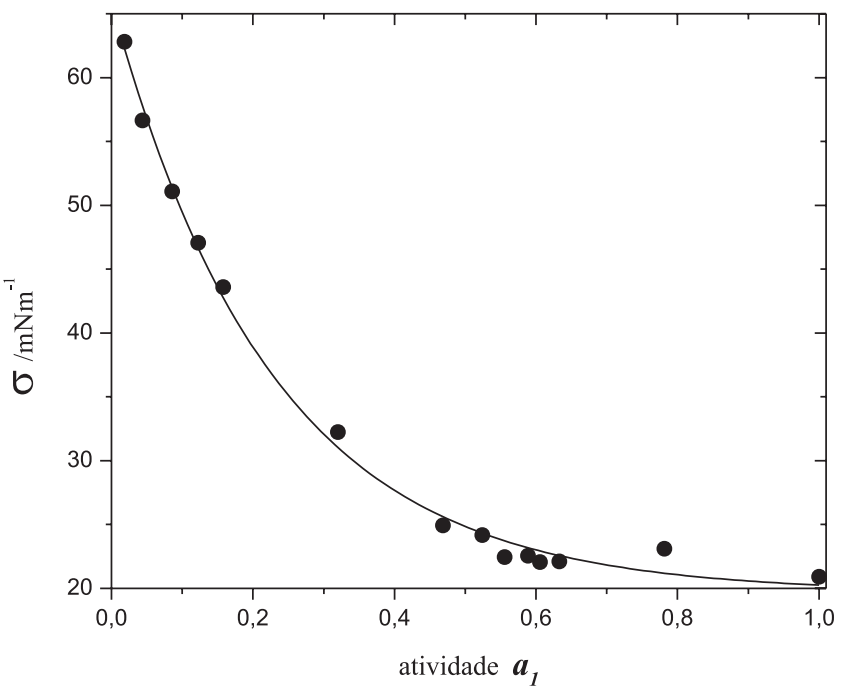

Figura 2. Gráfico da tensão superficial, $\sigma$, versus a atividade de n-propanol, $a_{1}$, para o sistema água n-propanol. A linha cheia representa o decaimento exponencial $\sigma=46,16 e^{-a_{1} /, 23}+19,69$

Os resultados das Figuras 1 e 2 podem ser apresentados na Figura 3. Na Figura 3 a percebe-se que a região de inflexão na curva atividade versus fração molar também está presente na curva tensão superficial versus fração molar. Esse comportamento representa a perda da idealidade da solução e o início da formação de agregados.

Para o cálculo do excesso de álcool na superfície deriva-se a tensão superficial com relação à atividade do álcool, cujo resultado é $\left(d \sigma / d a_{1}\right)_{T}=-\frac{k}{k^{\prime}} e^{-a_{1} / k^{\prime}}$.

Substituindo essa derivada na Equação 2, resulta

$$
\Gamma_{1}^{2}=\frac{k a_{1}}{k^{\prime} R T} e^{-a_{1} / k^{\prime}}
$$

A isoterma de adsorção é obtida pelo gráfico do excesso su- 


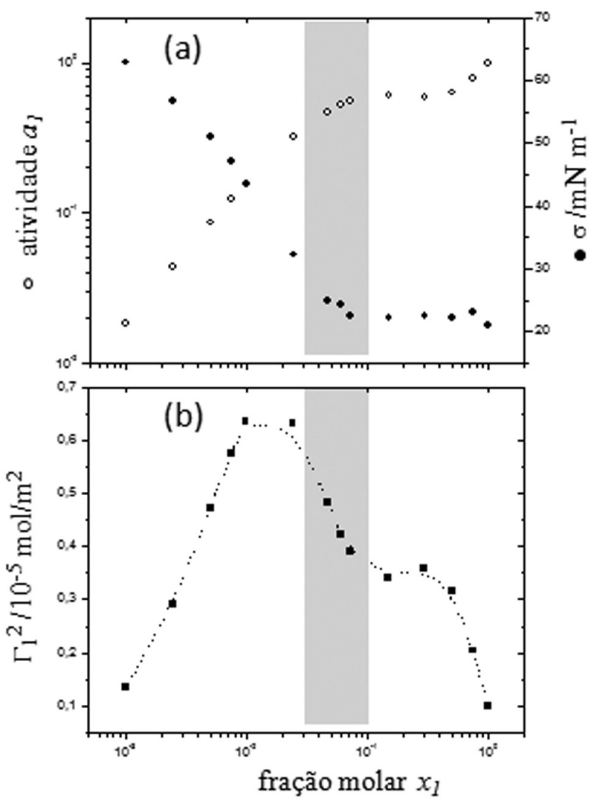

Figura 3. (a) Atividade, $a_{1}(\mathrm{O})$, e tensão superficial, $\sigma(\bullet)$, em função da fração molar de n-propanol, $x_{1}$, para o sistema água n-propanol; (b) isoterma de adsorção para o sistema água n-propanol, representada pelo grau de excesso superficial, $\Gamma_{1}^{2}$, em função da fração molar do álcool, $x_{1}$. A área em destaque reflete a perda de percolação das pontes de hidrogênio

perficial, calculado pela Equação 5, em função da fração molar do álcool, Figura 3b. Como a tensão superficial diminui com o aumento da concentração do álcool percebe-se, nessa figura, o aumento do excesso superficial até o recobrimento da monocamada. Após a região de máximo, $x_{1}$ em torno de 0,02 , tem-se uma diminuição do excesso superficial que coincide com o início da formação de agregados no seio da solução. Ou seja, a metodologia utilizada permite reproduzir os resultados apresentados no trabalho de Yano, que mostram que, a partir de uma dada concentração de álcool na solução, ocorre o decréscimo no excesso de superfície e a formação de agregados moleculares no seio da solução.

Os resultados para o sistema água etanol constam da Figura 4. Com a diminuição da tensão superficial com o aumento da fração molar do etanol, Figura 4a, a isoterma na Figura 4b apresenta um máximo em torno de $x_{1}=0,04$, valor muito próximo ao obtido por reflexão de nêutrons, ${ }^{25}$ em 0,01 . Os parâmetros da interação binária para água(2) etanol(1) são $A_{12}=1,6798$ e $A_{21}=0,9227$. A metodologia também foi aplicada ao sistema água(2) $n$-butanol(1), com $A_{12}$ $=1,0996$ e $A_{21}=4,176$. Esse último sistema apresenta miscibilidade parcial, com separação de fases em $x_{1}=0,018$ a $25^{\circ} \mathrm{C}$. Sua isoterma é apresentada na Figura 5, aonde se percebe o rápido aumento do excesso superficial com o aumento da concentração do álcool.

\section{CONCLUSÕES}

Isotermas de adsorção de misturas binárias formadas por água e alcoóis simples podem ser introduzidas como atividade de experimentação teórico-práticas de físico-química para os cursos de química, com o emprego de uma metodologia simples e eficiente como o método da contagem das gotas. Após a parte experimental, todo o tratamento dos dados pode ser empregado para desenvolver habilidades como o trabalho em planilhas eletrônicas e ajuste de função, bem como a construção de gráficos no computador. Conceitos como tensão superficial, excesso superficial, agregados moleculares, atividade e idealidade de soluções, por exemplo, são abordados dentro dessa aula
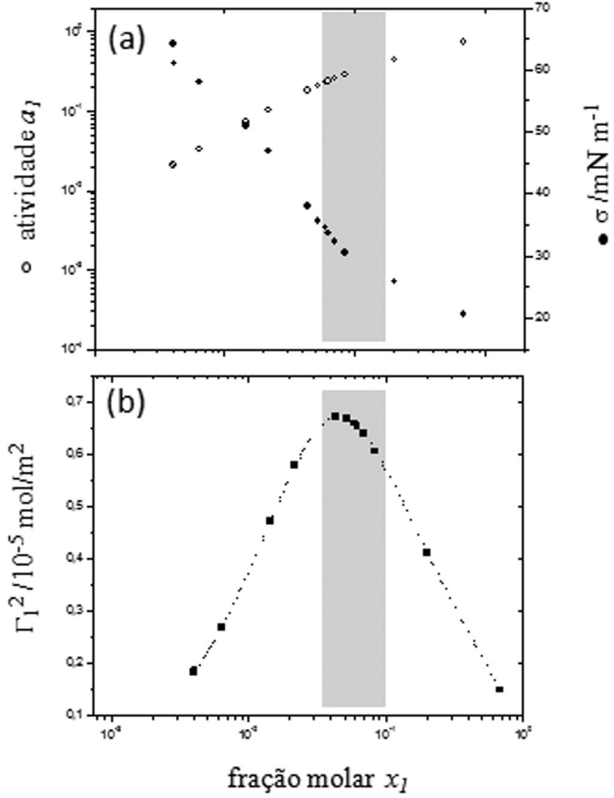

Figura 4. (a) Atividade, $a_{1}(\mathrm{O})$, e tensão superficial, $\sigma(\bullet)$, em função da fração molar de n-propanol, $x_{1}$, para o sistema água etanol; (b) isoterma de adsorção para o sistema água etanol, representada pelo grau de excesso superficial, $\Gamma_{I}^{2}$, em função da fração molar do álcool, $x_{l}$. A área em destaque reflete a perda de percolação das pontes de hidrogênio

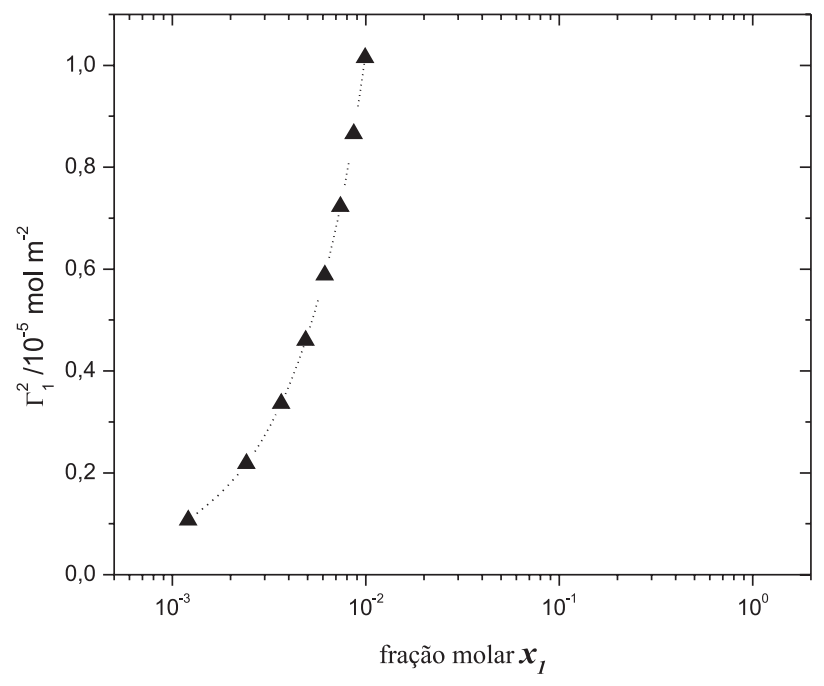

Figura 5. Isoterma de adsorção para o sistema água n-butanol, representada pelo grau de excesso superficial, $\Gamma_{1}^{2}$, em função da fração molar do álcool, $x_{1}$

para interpretar as isotermas de adsorção em misturas binárias de água e álcool. Considerando os equipamentos empregados nessa atividade de experimentação, essas isotermas obtidas são quali e quantitativamente comparáveis às determinadas com metodologias mais precisas.

\section{MATERIAL SUPLEMENTAR}

Apresenta uma proposta de roteiro para aula experimental. Esse material encontra-se disponível em http://www.quimicanova.sbq.org. br, na forma de arquivo PDF, com acesso livre.

\section{AGRADECIMENTOS}

Ao CNPq e à FUNAPE pelo suporte financeiro. 


\section{REFERÊNCIAS}

1. de Oliveira, A. E.; Soares, M. H. F. B.; de Souza, A. R.; Educación Química 2006, 17, 114 .

2. http://www.dsm.com/en_US/html/dep/painting_process.htm, acessada em Agosto 2009.

3. Amaral, M. H.; das Neves, J.; Oliveira, A. Z.; Bahia, M. F.; J. Surfactants Deterg. 2008, 11, 275; Harkot, J.; Janczuk, B.; Appl. Surf. Sci. 2009, 255, 3623

4. Keng, P. S.; Basri, M.; Zakaria, M. R. S.; Rahman, M. B. A.; Ariff, A. B.; Rahman, R. N. Z. A.; Salleh, A. B.; Industrial Crops and Products 2009, 29, 37.

5. Kieweg, S. L.; Katz, D. F.; J. Biomechanical Engineering-Transactions of the Asme 2006, 128, 540 .

6. Doll, K. M.; Moser, B. R.; Ethan, S. Z.; Energy Fuels 2007, 21, 3044.

7. Eissa, A. M. F.; El-Sayed, R.; Grasas y Aceites 2006, 57, 367.

8. Bezeigues, J. B.; Serieye, S.; Crosset-Perrotin, L.; Leser, M. E.; Colloids Surf., A 2008, 331, 56.

9. Carter, D. L.; Shah, D. O.; J. Surfactants Deterg. 2005, 8, 91.

10. Choi, C. Q.; Scientific American 2008, 299, 32.

11. Wakisaka, A.; Ohki, T.; Faraday Discuss. 2005, 129, 231; Nishi, N.; Yamamoto, K.; J. Am. Chem. Soc. 1987, 109, 7353.

12. Soper, A. K.; Dougan, L.; Crain, J.; Finney, J. L.; J. Phys. Chem. B 2006, 110, 3472; Dougan, L.; Bates, S. P.; Hargreaves, R.; Fox, J. P.; Crain, J.; Finney, J. L.; Reat, V.; Soper, A. K.; J. Chem. Phys. 2004, 121, 6456.
13. Nishikawa, K.; Kodera, Y.; Iijima, T.; J. Phys. Chem. 1989, 93, 6559.

14. Egashira, K.; Nishi, N.; J. Phys. Chem. B 1998, 102, 4054.

15. Fileti, E. E.; Chaudhuri, P.; Canuto, S.; Chem. Phys. Lett. 2004, 400, 494.

16. Kusalik, P. G.; Lynbartsev, A. P.; Bergman, D. L.; Laaksonen, A.; J. Phys. Chem. B 2000, 104, 9526.

17. Yano, Y. F.; J. Colloid Interface Sci. 2005, 284, 255.

18. Adamson, A. W.; Gast, A. P.; Physical Chemistry of Surface, $6^{\text {th }}$ ed., John Wiley \& Sons: New York, 1997, cap. 2.

19. Ewart, H. A.; Hyde, K. E.; J. Chem. Educ. 1992, 69, 814; Behring, J. L.; Lucas, M.; Machado, C.; Barcellos, I. O.; Quim. Nova 2004, 27, 492; Teixeira Neto, E.; Malta, M. M.; dos Santos, R. G.; Quim. Nova 2009, $32,223$.

20. Tate, T.; Philos. Mag. 1864, 27, 176.

21. van Laar, J. J. Z.; Phys. Chem. 1929, 72, 723.

22. Seader, J. D.; Siirola, J. J.; Barnicki, S. D. Em Perry's Chemical Engineers' Handbook; Perry, R. H.; Green, D. W.; Maloney, J. O., eds.; $7^{\text {th }}$ ed., Mc-Graw-Hill: New York, 1997, cap. 13.

23. http://www.broffice.org, acessada em Dezembro 2009 e Junho 2010.

24. Hoffman, J. M. L.; Avaliar para Promover: As Setas do Caminho, Mediação: Porto Alegre, 2001.

25. Li, Z. X.; Lu, J. R.; Styrkas, D. A.; Thomas, R. K.; Rennie, A. R.; Penfold, J.; Mol. Phys. 1993, 80, 925. 


\section{ADSORÇÃO E PROPRIEDADES DE VOLUME DE MISTURAS BINÁRIAS ÁGUA ÁLCOOL: UM EXPERIMENTO DIDÁTICO COM BASE EM MEDIDAS DE TENSÃO SUPERFICIAL}

Michelly C. dos Santos, Aline P. Moraes, Maykon A. Lemes, Emília C. D. Lima e Anselmo E. de Oliveira*

Instituto de Química, Universidade Federal de Goiás, CP 131, 74001-970 Goiânia - GO, Brasil

1. Procedimento Experimental

a. Prepare as soluções de água e etanol nas proporções indicadas na Tabela 1;

b. Determine as densidades das soluções, assim como a da água e a do álcool puro;

c. Determine as tensões superficiais das soluções, bem como para água e o álcool puro, em duplicatas.

I. Prender uma bureta/pipeta pequena em um suporte.

II. Encher a bureta com uma das soluções.

III. Determinar o número de gotas que se formam por $\mathrm{mL}$.

2. Tratamento dos Dados

a. Monte a planilha para tratamento dos dados.

I. Insira a fração molar do álcool, $\mathrm{x}_{1}$, para cada uma das soluções.

II. Calcule a $\ln \left(\mathrm{x}_{1}\right)$.

III. Insira as densidades das soluções, $\rho$.

IV. Insira o número de gotas.

V. Calcule a tensão superficial da solução, $\sigma$.

VI. Calcule a $\ln \left(\gamma_{1}\right)$ com base na equação de van Laar.
VII. Calcule o coeficiente de atividade, $\gamma_{1}$.

VIII. Calcule a atividade do álcool, $a_{1}$, sabendo que $a$ $=\gamma x$.

b. Faça o gráfico da atividade do álcool, $a_{l}$, em função da sua fração molar, $x_{l}$, e interprete esse resultado.

c. Se preferir, faça o gráfico de $a_{1}$ versus $\ln \left(x_{1}\right)$ e interprete esse resultado.

d. Faça o gráfico da tensão superficial, $\sigma$, em função da atividade do álcool, $a_{l}$, e interprete esse resultado.

e. Faça o ajuste exponencial. No OpenOffice Calc isso é feito ao inserir um gráfico e, em seguida, inserir linha de tendência.

f. Se preferir, faça um gráfico de $\sigma$ versus $\ln \left(x_{1}\right)$, e interprete esse gráfico.

g. Insira mais duas colunas à direita da planilha.

i. Calcule a derivada da função exponencial $\sigma=k e^{-a_{1} / k^{\prime}}+k^{\prime \prime}$ para cada valor de $a_{1}$.

ii. Calcule o excesso superficial, $\Gamma_{1}^{2}$, para cada solução.

h. Faça o gráfico do excesso superficial, $\Gamma_{1}^{2}$, em função do $\ln \left(x_{1}\right)$, e interprete essa isoterma.

Tabela 1 - Composição das soluções para o sistema água $n$-propanol.

\begin{tabular}{lccccccccccccc}
\hline $\mathrm{n}$ & 1 & 2 & 3 & 4 & 5 & 6 & 7 & 8 & 9 & 10 & 11 & 12 & 13 \\
\hline $\mathrm{x}_{1} / 10^{-2}$ & 101 & 248 & 503 & 744 & 990 & 2,433 & 4,667 & 6,118 & 7,373 & 15,159 & 30,077 & 50,050 & 75,153 \\
\hline
\end{tabular}

